\title{
Investigation of gene expression profiles in a rat adjuvant arthritis model suggests an effective role of triptolide via PI3K-AKT signaling
}

\author{
YANG ZOU and WEIFENG HU \\ Department of Orthopedics, The Second Affiliated Hospital of Zhejiang Chinese Medical University, \\ Hangzhou, Zhejiang 310005, P.R. China
}

Received August 28, 2018; Accepted March 13, 2019

DOI: $10.3892 /$ etm.2019.7425

\begin{abstract}
Rheumatoid arthritis (RA) is a common systemic autoimmune disease mainly involving the formation of a synovial pannus, for which no effective treatment is available. In order to study the molecular biological mechanisms underlying the inhibition of RA synovial pannus by triptolide, differentially expressed genes in synovial tissues from an adjuvant arthritis (AA) rat model with and without triptolide treatment were detected in an mRNA microarray profile produced by Agilent Technologies and verified by reverse transcription-quantitative polymerase chain reaction analysis (RT-qPCR). An AA model was established by subcutaneously injecting $0.1 \mathrm{ml}$ Freund's complete adjuvant daily for 18 days and scored by arthritis index assessment. Subsequently, triptolide $(0.4 \mathrm{mg} / \mathrm{kg})$ or an equivalent amount of saline was administered daily for 14 days. At the end of the experiment, synovial tissues were obtained from the ankle joints of the rats' hind legs. Total RNA was extracted and purified, and microarray hybridization was used to obtain the gene expression profile for RA with and without triptolide treatment. A total of 48 genes were identified to be differentially expressed between the treatment and model groups, including 32 upregulated and 16 downregulated genes. The possible signaling pathways associated with the effect of triptolide were investigated by Gene Ontology and pathway analysis, revealing that the phosphoinositide-3 kinase (PI3K)/AKT signaling pathway has a key role in the proliferation and apoptosis of synovial cells in RA joints. Reverse transcription-quantitative polymerase chain reaction analysis was applied to confirm the aberrant expression of key mRNAs and revealed that vascular endothelial
\end{abstract}

Correspondence to: Mr. Weifeng Hu, Department of Orthopedics, The Second Affiliated Hospital of Zhejiang Chinese Medical University, 318 Chaowang Road, Hangzhou, Zhejiang 310005, P.R. China

E-mail: zjwork2007@sina.com

Key words: triptolide, rheumatoid arthritis, adjuvant arthritis, gene chip growth factor (VEGF) A and C1q and tumor necrosis factor related protein 3 (C1QTNF3) were downregulated in the treatment group compared with the model group $(\mathrm{P}<0.05)$. In conclusion, triptolide may exert its effects against RA via the PI3K/AKT pathway and has an inhibitory effect on the expression of VEGFA and C1QTNF3, thus are potentially associated with the occurrence and development of RA.

\section{Introduction}

Rheumatoid arthritis (RA) is a common systemic, non-infectious autoimmune disease that is mainly characterized by synovitis. The pathological characteristics of RA include autoimmune response and chronic inflammation-induced synovial hyperplasia, angiogenesis and cartilage destruction (1,2). In Europe, a preliminary understanding of this disease entity prevailed as early as the 17th century, but it was not until 1859, when it was named RA by Garrod, that it was more comprehensively understood $(3,4)$. The incidence rate of RA in China is $\sim 30 \%$ ( $\sim 4$ million patients) and the disability rate $(60 \%)$ is relatively high (5). One of the most important pathophysiological processes is the formation of the synovial pannus, which erodes and destroys adjacent articular cartilage and bone, resulting in joint remodeling, distortion and ankylosis (6). There is currently no effective treatment available for this common disease, and the underlying mechanisms of the exited targeting drug remain elusive.

Triptolide is a non-steroidal anti-inflammatory agent and immunosuppressant. Previous clinical studies have reported that triptolide possesses anti-rheumatic, anti-oxidative and anti-cancer properties, and is the most commonly used Chinese medicine in the treatment of RA. Triptolide, the major active component of Tripterygium wilfordii, is a diterpene lactone epoxide compound whose specific molecular targets in RA have remained to be fully determined (7-9). Only few studies have assessed the mechanisms and pathways associated with the action of triptolide. In order to further elucidate the therapeutic mechanisms and targets of triptolide in RA, the present study used a rat model of RA to establish a basis for clinical treatment and to provide fundamental evidence supporting the use of this Traditional Chinese Medicine (TCM) component in the treatment of RA $(10,11)$. 
Many human infectious diseases are typically associated with the deregulation of gene expression, and even normal tissues are accompanied by changes in gene expression patterns (12). Gene chip analysis is a milestone technological innovation in the field of molecular biology and one of the most advanced and effective methods. It allows researchers to determine how thousands of genes act simultaneously, quantify certain genes in patients to identify a disease and provides novel targets for drug design and disease treatment $(13,14)$. However, the application of gene chip technology to assess the treatment effect of triptolide in RA has not been previously reported. By analyzing the differences in the gene expression profile in synovial tissues of model animals with and without drug administration, the differential gene expression spectrum of synovial tissue in RA may be established and the mechanism of the triptolide treatment effect on RA may be qualitatively and quantitatively analyzed at the gene level.

The aim of the present study was to determine the differentially expressed genes (DEGs) in synovial tissues of RA rats by using the gene chip technique and reverse transcription-quantitative polymerase chain reaction (RT-qPCR), and to explore the targets of the DEGs following treatment of RA by triptolide, including the direct molecular targets of triptolide and the effects of RA declining as a result of triptolide treatment.

\section{Materials and methods}

Animals. A total of 20 pathogen-free male adult Wistar rats (age, 8 weeks; weight, $150 \pm 10 \mathrm{~g}$ ) were provided by the Shanghai Experimental Animal Center of the Chinese Academy of Sciences/Shanghai Shrek Experimental Animal Co., Ltd. [animal certificate no. SCXK (Shanghai 2007-0005)]. All of the rats were housed at the Animal Center of Zhejiang Chinese Medical University (Hangzhou, China) at a constant temperature of $23 \pm 1^{\circ} \mathrm{C}$, humidity of $40-70 \%$ and under a 12-h light/dark cycle, with free access to food and water. All procedures conformed to the guidelines of the National Institutes of Health Guide for the Care and Use of Laboratory Animals and were approved by the Ethics Committee for the Use of Experimental Animals of Zhejiang Chinese Medical University (Hangzhou, China; animal permit no. SYXK (Zhejiang Province 2008-0115).

Experimental groups and adjuvant arthritis (AA) model. The 20 rats used in the present study were randomly divided into two groups, namely the AA model group and the triptolide treatment (TP) group ( $\mathrm{n}=10$ per group). The AA model was induced by daily injecting Freund's complete adjuvant $(0.1 \mathrm{ml})$ into the muscle of the hind paw with a microsyringe for $\sim 18$ days. The activity of the animals and ankle swelling were observed $(10,15)$. At day 18, the successful establishment of this model was evaluated by determining the arthritis index (AI) $(16,17)$. The standard grading of AI was as follows: 0, no redness; 1, mild swelling of the toe joint, inflammation in a single area of the paw or foot pad; 2 , mild redness of the joint, inflammation in $>2$ areas of the paw and foot pad or ankle; 3 , moderate redness and mild dysfunction of the joint; 4 , severe redness of the joint, rigidity or even malformation, severe dysfunction. Each paw scored 4 points, adding up to a total of 8 points. The model was considered to be successfully established if the AI score was $>4$ points $(16,17)$.
Drug administration and sample collection. After model establishment, the rats in the TP group were administered the medicine from the 18th day onwards as follows: First, the foot pad of the hind leg was routinely sterilized, and each rat received a muscle injection with triptolide at a dose of $0.4 \mathrm{mg} / \mathrm{kg}$ daily for 14 days $(18,19)$. The rats in the model group were injected with an equal volume of $0.9 \%$ physiological saline. All of the rats were sacrificed on day 14, and the hind leg and ankle joint were resected and processed. Following anesthesia by intraperitoneal injection of $10 \%$ chloral hydrate $(400 \mathrm{mg} / \mathrm{kg})$ and after disinfection with alcohol, the skin was incised along the middle of the ankle joint until an area $\sim 2 \times 2 \mathrm{~cm}$ was exposed. The joint cavity was opened, and the synovial tissue around the ankle was carefully resected with a surgical blade. The synovial tissue specimen was rinsed with diethyl pyrocarbonate, added into a cryopreservation tube and placed in liquid nitrogen to be used for gene chip detection and qPCR analysis $(20,21)$. The rats were sacrificed by dislocation of the neck at 30 min after anesthesia.

Total RNA extraction and purification. Total RNA was extracted with TRIzol reagent (Invitrogen; Thermo Fisher Scientific, Inc., Waltham, MA, USA); 50-100 mg tissue/ml TRIzol was used in the present study and all procedures were performed according to the manufacturer's protocol. Quality control of the RNA samples was performed with a Nanodrop ND-1000 spectrophotometer (Thermo Fisher Scientific, Inc.) by measuring the absorbance of each sample at $260 \mathrm{~nm}(21)$. The RNA samples were then subjected to $1 \%$ agarose gel electrophoresis. In order to obtain high-quality purified RNA samples, and reach the labeling efficiency of the probe and optimal results of hybridization in the subsequent experiment, the Qiagen RNeasy kit (Qiagen GmBH, Hilden, Germany) was used to purify total RNA, according to the manufacturer's protocol $(22,23)$.

RNA labeling and microarray hybridization. The mRNA microarray profile (ID: 014879) was provided by Agilent Technologies, Inc. (Santa Clara, CA, USA). Total RNA was amplified and labeled using the Low-Input Quick Amp Labeling kit, One-Color (cat. no. 5190-2305; Agilent Technologies, Inc.), according to the manufacturer's protocol. Labeled complementary RNA (cRNA) was purified with the RNeasy mini kit (cat. no. 74106; Qiagen GmBH). Each slide was hybridized with $1.65 \mu \mathrm{g}$ cyanine 3-labeled cRNA using the Gene Expression Hybridization kit (cat. no. 5188-5242; Agilent Technologies, Inc.) in a hybridization oven (cat. no. G2545A; Agilent Technologies, Inc.) according to the manufacturer's protocol. After $17 \mathrm{~h}$ of hybridization, the slides were washed in staining dishes (cat. no. 121; Shandon ${ }^{\mathrm{TM}}$; Thermo Fisher Scientific, Inc.) with a Gene Expression Wash Buffer kit (cat. no. 5188-5327; Agilent Technologies, Inc.) according to the manufacturer's protocol $(24,25)$. The microarray data were deposited in the GEO repository (https://www.ncbi.nlm.nih. gov/geo/query/acc.cgi?acc=GSE124639).

Screening of DEGs. The raw microarray data were processed using GeneSpring software version 12.6.1 (Agilent Technologies, Inc.). The data were normalized and the gene expression was compared between the two groups. The gene 
chip data were screened for DEGs according to different selection criteria (fold-change $>2$ for upregulated and $<0.5$ for downregulated genes; $\mathrm{P}<0.01)$.

Gene ontology (GO) and pathway analysis. The GO (http://www.geneontology.org) database was used to identify functional terms enriched by the DEGs. GO analysis determines the accumulation of DEGs in terms of three independent categories, namely biological process, cellular component and molecular function. The DEGs are used as input in the GO analysis. In the analysis of DEGs from gene expression profiles, GO may be used to provide information on the functions, structural characterization and gene function classification labeling.

Generation of the interaction network. The pathway analysis was performed using the and Kyoto Encyclopedia of Genes and Genomes (KEGG) database (http://www.genome.jp/kegg), which provides a systematic analysis of gene function, associated genome information and functional information. Cell biochemical processes, including membrane transport, metabolism, cell cycle and signal transmission, may be fully elucidated by searching the more advanced KEGG pathway database. Each node in the database was mapped to calculate the number of genes per node.

$R T$ - $q P C R$. Total RNA samples from each group were reverse-transcribed into complementary (c)DNA. RT was performed with the Maxima First Strand cDNA Synthesis kit for RT-qPCR (cat. no. K1641; Thermo Fisher Scientific, Inc.). qPCR analysis was performed using the DyNAmo Flash SYBR Green qPCR kit (cat. no. F415XL; Thermo Fisher Scientific, Inc.) to evaluate vascular endothelial growth factor (VEGF) A and Clq and tumor necrosis factor related protein 3 (C1QTNF3) levels using $\beta$-actin as the internal control. Each sample was analyzed in triplicate. The PCR consisted of an initial denaturation step at $94^{\circ} \mathrm{C}$ for $5 \mathrm{~min}$, followed by an amplification with 35 reaction cycles, including denaturation at $94^{\circ} \mathrm{C}$ for $30 \mathrm{sec}$, annealing at $58^{\circ} \mathrm{C}$ for $30 \mathrm{sec}$, and final extension at $72^{\circ} \mathrm{C}$ for $1 \mathrm{~min}$ (26). The relative mRNA expression levels were calculated using the quantification cycle $(\mathrm{Cq})$ method. The primers used were as follows: VEGFA, forward 5'-GAGGAAAGGGAAAGGGTCAAA-3' and reverse 5'-CAC AGTGAACGCTCCAGGATT-3'; C1QTNF3, forward 5'-GTG CTCAGAAATAATTGGCTCCT-3' and reverse 5'-AAGGTG TGGCAAGCCAAATG-3'; and $\beta$-actin, forward 5'-TCTGTG TGGATTGGTGGCTCTA-3' and reverse 5'-CTGCTTGCT GATCCACATCTG-3'.

Statistical analysis. SPSS 17.0 software (SPSS Inc., Chicago, IL, USA) was used for statistical analysis. One-way analysis of variance and the Student-Newman-Keuls test were used to analyze the differences between the experimental groups. Values are expressed as the mean \pm standard deviation. $\mathrm{P}<0.05$ was considered to indicate a statistically significant difference.

\section{Results}

AI score. The AI scores in the rats of the present study was determined to confirm successful model establishment.



Figure 1. Comparison of AI scores between groups. ${ }^{*} \mathrm{P}<0.05$, vs. all other groups/time-points. Data are expressed as the mean \pm standard deviation $(\mathrm{n}=10)$. AI, arthritis index; TP, triptolide.

AI scores of $>4$ on the 18th day after modeling were considered to indicate successful establishment of the model. A comparison of the AI scores in the model and TP groups is presented in Fig. 1. In the TP group, the AI score was significantly decreased following TP treatment, while that in the model/vehicle-treated group was unchanged.

Screening of DEGs between the treatment and model groups. The gene chip data were screened according to the abovementioned fold change/significance criteria for DEGs. A number of DEGs were screened out presented in the list, the data of which are presented in Fig. 2. There were 6 upregulated DEGs and 5 downregulated DEGs listed, including VEGFA and C1QTNF3.

GO analysis. The DEGs were subjected to a GO functional enrichment analysis. It was revealed that the DEGs were implicated in a wide range of molecular functions, including tumor-associated pathways, cell cycle regulation and interaction between cell receptors and extracellular matrix. Details of the association between RA and the GO functional terms associated with the genes affected by triptolide treatment in RA are presented in Fig. 3. The top GO terms include response to redox state, regulation of dendritic spine morphogenesis and establishment of tissue polarity.

Pathway analysis. Using KEGG pathway enrichment analysis, the DEGs were identified to participate in various signaling pathways. The detailed information is presented in Fig. 4. The most significant pathways are listed in the top panel of the figure. The pathway analysis suggested that the phosphoinositide-3 kinase (PI3K)/AKT signaling pathway has a key role in the proliferation and apoptosis of synovial cells of RA joints. Furthermore, other pathways were listed that could be potentially involved in the whole regulation.

RT-qPCR analysis. The distinct DEGs VEGFA and C1QTNF3 were selected from the microarray and RT-qPCR was used for detection using the same RNA samples. Thereby, the results obtained with the gene chip method were verified. The mRNA 


\begin{tabular}{lllc}
\hline Gene symbol & Gene bank ID & Gene name & Fold-change \\
\hline Tyrp1 & NM_001106664 & Tyrosinase-related protein 1 & 4.43 \\
\hline Olr757 & NM_001000613 & Olfactory receptor 757 & 4.36 \\
\hline Pcdhga9 & NM_001037158 & Protocadherin gamma subfamily A, 9 & 4.12 \\
\hline Wnt6 & NM_001108226 & $\begin{array}{l}\text { Wingless-type MMTV integration site } \\
\text { family, member 6 }\end{array}$ & 3.68 \\
\hline Il1b & NM_031512 & Interleukin 1 beta & 3.52 \\
\hline Hnf4a & NM_022180 & Hepatocyte nuclear factor 4, alpha & 3.40 \\
\hline Zbtb16 & XM_006243016 & Zinc finger and BTB domain containing 16 & 0.31 \\
\hline Vegfa & NM_031836 & Vascular endothelial growth factor A & 0.29 \\
\hline LOC10255539 & XR_355802 & Zinc finger protein 16-like & 0.29 \\
\hline Clqtnf3 & NM_001134436 & C1q and tumor necrosis factor related protein 3 & 0.24 \\
\hline Adamts19 & XM_006254755 & $\begin{array}{l}\text { ADAM metallopeptidase with } \\
\text { thrombospondin type 1 motif, 19 }\end{array}$ & 0.19 \\
\hline
\end{tabular}

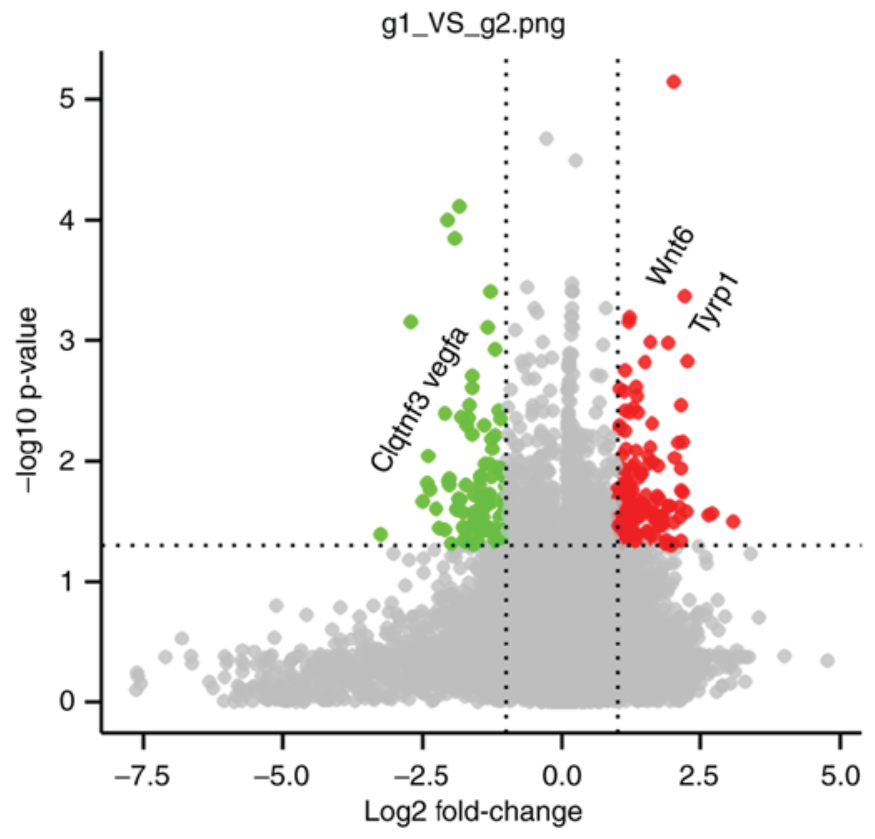

Figure 2. DEGs in the joints of triptolide- vs. vehicle-treated rats with rheumatoid arthritis. The gene chip data were screened according to the screening criteria of DEGs (fold-change $>2$ for upregulated and $<0.5$ for downregulated genes). Among the significantly upregulated DEGs are TYRP1, OLR757, PCDHGA9, WNT6, IL1Band HNF4A, while ZBTB16, VEGFA, LOC10255539, C1QTNF3 and Adamts19 are among the downregulated DEGs. DEG, differentially expressed gene; VEGFA, vascular endothelial growth factor A; C1QTNF3, C1q and tumor necrosis factor related protein 3.

expression of VEGFA (27) and C1QTNF3 (28) was significantly downregulated in the TP treatment group compared with that in the vehicle-treated group $(\mathrm{P}<0.05 ;$ Fig. 5). The expression of VEGFA and C1QTNF3 in the vehicle (model) and TP groups was therefore consistent with that determined by gene chip detection.

\section{Discussion}

The present study investigated the effect of triptolide treatment on the gene expression profiles in the joint tissue of RA model rats. The major manifestations of RA are joint pain, edema, limited range of flexion and extension, morning stiffness, joint deformities and even joint swelling and burning (1). Since RA is a lingering disease that is associated with a high disability rate, the primary purpose of treatment is to relieve these symptoms and prevent the progression of the disease. TCM generally treats the body as a whole, applying the principles of syndrome differentiation $(29,30)$. It helps relieve the pain and enables functional recovery of patients with joint swelling and pain, and is associated with only few adverse reactions, effectively reducing the risk of disability (31).

It was previously demonstrated that triptolide significantly reduces paw edema induced by carrageenan or egg white in 


\begin{tabular}{|c|c|c|c|c|}
\hline GO_ID & GO_term & Category & TargetGene_in_GO & Pvalue \\
\hline GO:0051775 & Response to redox state & Biological_process & 2 & $3.43 \times 10^{-8}$ \\
\hline GO:0061001 & $\begin{array}{l}\text { Regulation of dendritic } \\
\text { spine morphogenesis }\end{array}$ & Biological_process & 2 & 4. $57 \times 10^{-6}$ \\
\hline GO:0007164 & $\begin{array}{l}\text { Establishment of tissue } \\
\text { polarity }\end{array}$ & Biological_process & 1 & $2.88 \times 10^{-5}$ \\
\hline GO:2000331 & $\begin{array}{l}\text { Regulation of terminal } \\
\text { buttom organization }\end{array}$ & Biological_process & 1 & $2.88 \times 10^{-5}$ \\
\hline GO:0016716 & Oxidoreductase activity & Molecular_function & 1 & $3.30 \times 10^{-5}$ \\
\hline GO:0001681 & $\begin{array}{l}\text { Sialate O-acetylesterase } \\
\text { activity }\end{array}$ & Molecular_function & 1 & 3. $30 \times 10^{-5}$ \\
\hline GO:0051879 & Hsp90 protein binding & Molecular_function & 2 & 4. $42 \times 10^{-5}$ \\
\hline GO:0060076 & Excitatory synapse & Cellular_component & 2 & $9.84 \times 10^{-5}$ \\
\hline GO:0030669 & $\begin{array}{l}\text { Clathrin-coated endocytic } \\
\text { vesicle membrane }\end{array}$ & Cellular_component & 1 & $1.08 \times 10^{-4}$ \\
\hline
\end{tabular}
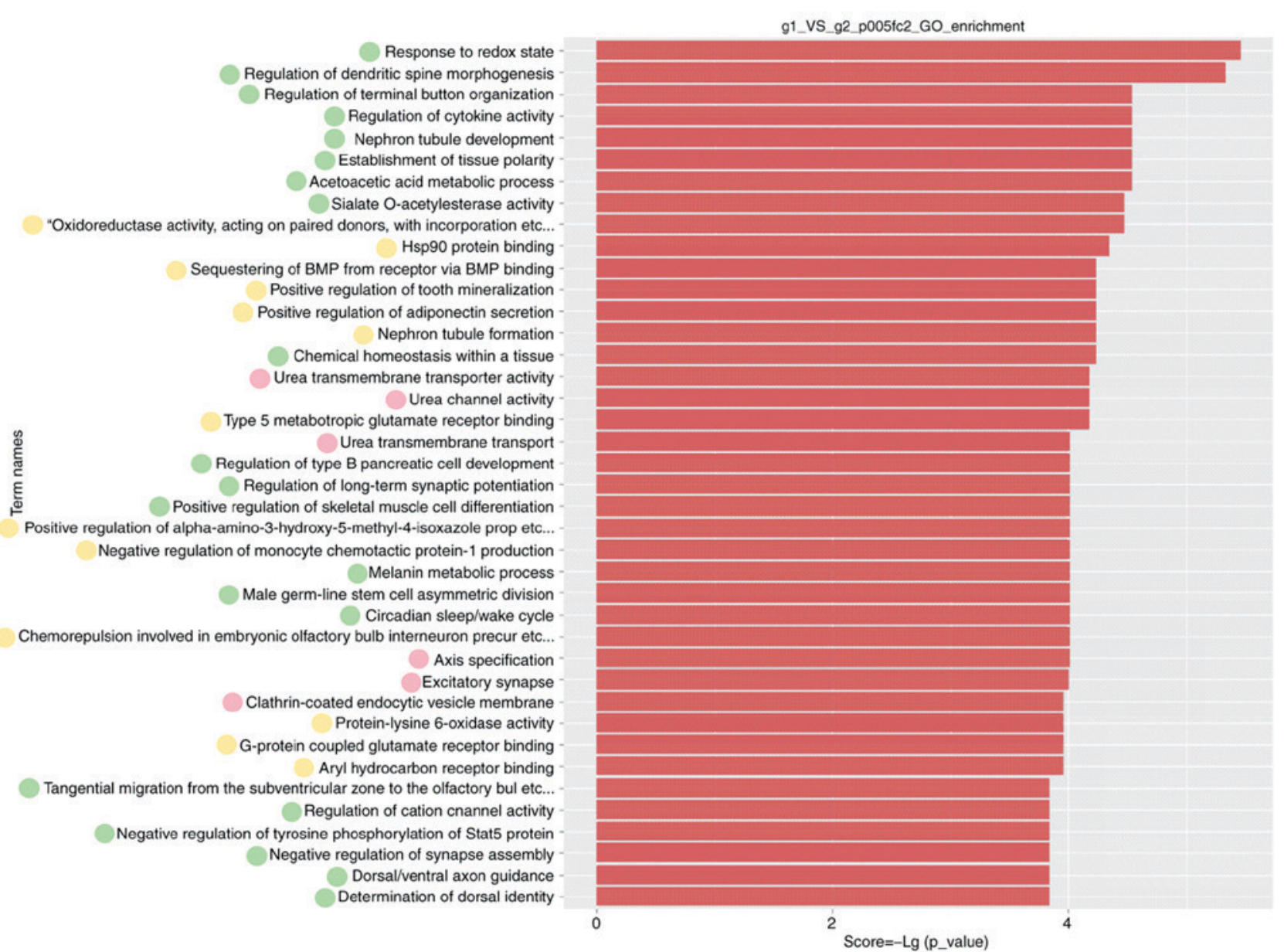

Figure 3. GO analysis. Details of the associations between rheumatoid arthritis and most relevant cellular or molecular processes are provided, based on the top GO terms in the list, including response to redox state, regulation of dendritic spine morphogenesis and establishment of tissue polarity. GO, gene ontology. Red indicator represents cellular component; green indicator represents biological process; yellow indicator represents molecular function. BMP, bone morphogenetic protein; HSP, heat shock proteins; STAT, signal transducer and activator of transcription.

AA rats. Its anti-inflammatory effects are mainly mediated by inhibiting the release of prostaglandins during the inflammatory response, and by decreasing the permeability of blood vessels and reducing the concentration of platelets, as well as the proliferation of fibers in the later stages of inflammation $(32,33)$. The AA model is an ideal animal model for investigating RA and screening of drugs, as its pathological changes and cellular immune abnormalities exhibit several 


\begin{tabular}{llcc}
\hline Pathway_ID & KEGG_name & TargetGene_in_this_pathway & Pvalue \\
\hline rno00053 & Ascorbate and aldarate metabolism & 1 & $5.037 \times 10^{-3}$ \\
\hline rno04151 & PI3K-Akt signaling pathway & 1 & $6.047 \times 10^{-3}$ \\
\hline rno00040 & Pentose and glucuronate & 1 & $7.881 \times 10^{-3}$ \\
\hline rno04310 & interconversions- & & $9.063 \times 10^{-3}$ \\
\hline rno00350 & Tyrosine metabolism & 1 & $1.0271 \times 10^{-2}$ \\
\hline rno04512 & ECM-receptor interaction & 1 & $4.668 \times 10^{-2}$ \\
\hline
\end{tabular}

Circadian rhythm-Rattus norvegicus (rat)

Glycosaminoglycan biosynthesis-heparan sulfate/heparin-Rattus no etc.. Maturity onset diabetes of the young-Rattus norvegicus (rat) Aacorbate and aldarate metabolism-Rattus norvegicus (rat) Melanogenesis-Rattus norvegicus (rat) Pentose and glucuronate interconversions-Rattus norvegicus (rat) Tyrosine metabolism-Rattus norvegicus (rat) Axon guidance-Rattus norvegicus (rat) Porphyrin and chlorophyll metabolism-Rattus norvegicus (rat) Hedgehog signaling pathway-Rattus norvegicus (rat) Starch and sucrose metabolism-Rattus norvegicus (rat) Drug metabolism-other enzymes-Rattus norvegicus (rat) Basal cell carcinoma-Rattus norvegicus (rat) Acute myeloid leukemia-Rattus norvegicus (rat) Protein processing in endoplasmic reticulum-Rattus norvegicus (rat) Cell adhesion molecules (CAMs)-Rattus norvegicus (rat) Pathways in cancer-Rattus norvegicus (rat) Metabolism of xenobiotics by cytochrome P450-Rattus norvegicus (rat) Retinol metabolism-Rattus norvegicus (rat) Drug metabolism-cytochrome P450-Rattus norvegicus (rat) Steroid hormone biosynthesis-Rattus norvegicus (rat) ECM-receptor interaction-Rattus norvegicus (rat) Chemical carcinogenesis-Rattus norvegicus (rat) Protein digestion and absorption-Rattus norvegicus (rat)
g1_VS_g2_p005fc2_KEGG_enrichment

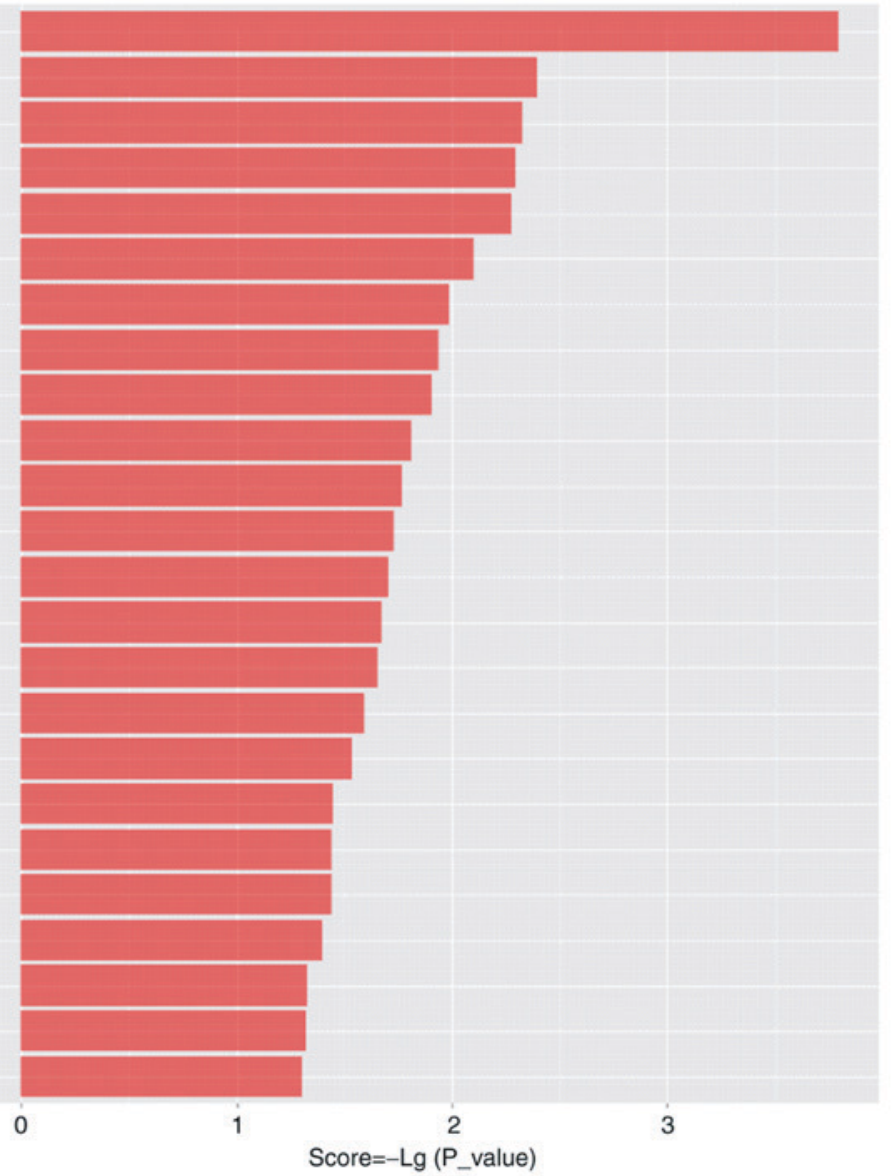

Figure 4. Pathway analysis. Each group of DEGs participated in the signal regulation pathway. The vehicle (model) and TP groups involved the signaling pathway were searched based on the KEGG library. The DEGs in the joints of triptolide- vs. vehicle-treated rats with rheumatoid arthritis were enriched in various KEGG signaling pathways. Pathway analysis suggested that the PI3K/AKT signaling pathway has a key role in the proliferation and apoptosis of synovial cells in RA joints, the DEGs in the joints of triptolide- vs. vehicle-treated rats with rheumatoid arthritis. rno, Rattus norvegicus; KEGG, Kyoto Encyclopedia of Genes and Genomes; PI3K, phosphoinositide-3 kinase; ECM, extracellular matrix.

similarities with RA (34). Therefore, AA rats were used as a model to study the effect of triptolide on the gene expression profile in joint tissues, which may provide an experimental basis for the treatment of RA in the future. However, one limitation was that the mechanisms of action of triptolide were not assessed via a comparison between RA model rats, triptolide-treated RA model rats and normal rats.
As the in vivo pathway and specific molecular mechanisms of action of triptolide in the prevention and treatment of RA have remained elusive, the present study investigated the pharmacological mechanism and effect of triptolide at the molecular level by employing the gene chip method. The present results identified distinct DEGs (VEGFA and C1QTNF3) that were selected from the microarray. It has 

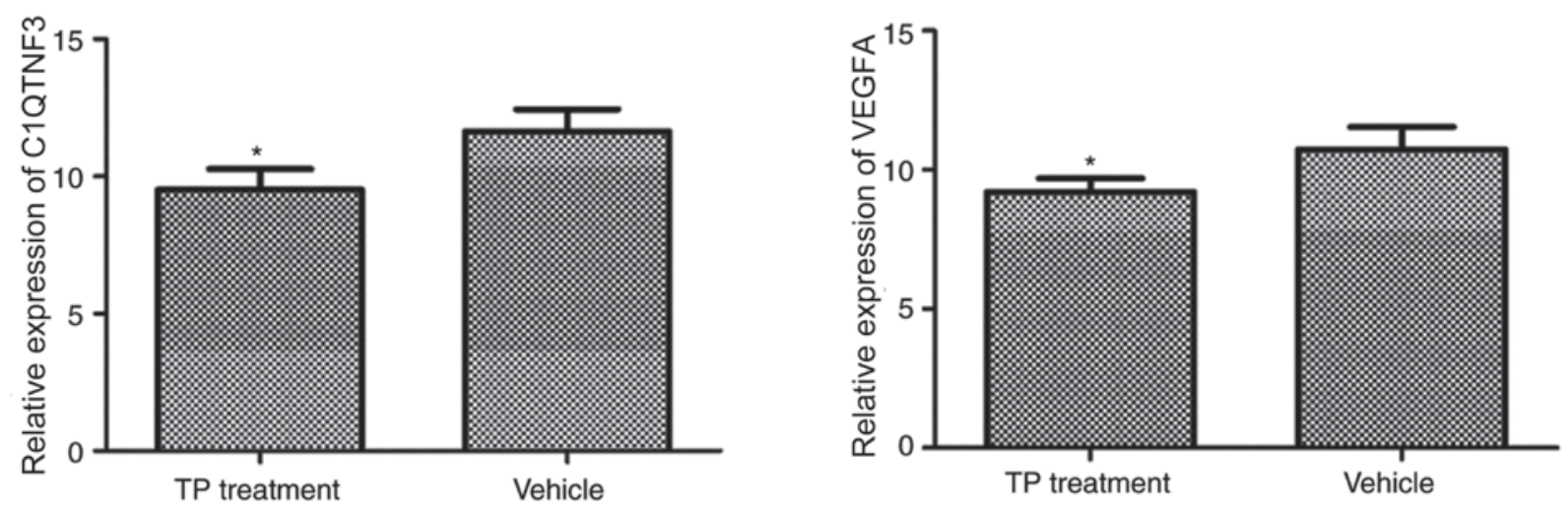

Figure 5. Reverse transcription-quantitative polymerase chain reaction analysis was used for verify the differentially expressed key mRNAs identified from the gene chip detection using the same RNA sample. The expression of VEGFA1 and C1QTNF3 in the vehicle (model) and TP groups was consistent with the results from the gene chip detection. The mRNA expression of VEGFA and C1QTNF3 was downregulated in the TP treatment group, and the difference from the model group was statistically significant. Values are expressed as the mean \pm standard deviation. ${ }^{*} \mathrm{P}<0.05$ vs. vehicle group. TP, triptolide; VEGFA, vascular endothelial growth factor A; C1QTNF3, C1q and tumor necrosis factor related protein 3.

been reported that activation of the PI3K/AKT pathway has a negative role in modulating genes that promote inflammation, thrombogenicity and vascular permeability, and thereby protect vascular function. Furthermore, the neuroprotective role of the PI3K/AKT pathway in other disease models, including cerebral ischemia, has been widely studied (35). In the present study, GO and KEGG pathway analysis suggested that the PI3K/AKT signaling pathway has a key role in the proliferation and apoptosis of synovial cells in RA joints with triptolide treatment. A previous study has revealed that an imbalance of the phosphatase and tensin homolog/PI3K/AKT pathway in rats with AA is one of the mechanisms of synovial neovasculization (36).

In the present study, the gene microarray profiles of the treatment and the model group were determined, from which the DEGs between the two groups were determined. The analysis demonstrated that triptolide affected the gene expression profile of AA model rats. A total of 48 DEGs were identified, among which 32 genes were upregulated and 16 were downregulated. The GO terms of these DEGs were determined, and included transcription factor regulation, kinase, cytoskeleton and protein synthesis, transport function, modification of cytokines, inflammatory response, immune response, cell proliferation and invasion and cell cycle regulation. The KEGG signaling pathways involved are mainly associated with angiogenesis, apoptosis, proliferation, oxidative stress and inflammation. The DEGs were determined to be involved in tumor-associated pathways, cell cycle regulation and cell receptor interaction with the extracellular matrix. According to the Bioinformatics analysis, certain significant DEGs, the key genes, identified from the microarray results were associated with tumor-like properties, and the genes located at the key signaling pathway nodes were determined. Two genes, VEGFA and C1QTNF3, were selected for verification of their expression levels by RT-qPCR. The results demonstrated that the expression of the two genes in the treatment group was lower compared with that in the model group, which was consistent with the results of the microarray, indicating that the reproducibility and reliability of the experiment was satisfactory.

\section{Acknowledgements}

Not applicable.

\section{Funding}

The present study was supported by Zhejiang Provincial Natural Science Foundation of China (grant no. LY14H060005).

\section{Availability of data and materials}

The datasets generated and analyzed during the current study are available in the GEO repository (https://www.ncbi.nlm. nih.gov/geo/query/acc.cgi?acc=GSE124639) and from the corresponding author on reasonable request.

\section{Authors' contributions}

$\mathrm{YZ}$ and WH established the animal model, calculated the arthritis index score and performed drug administration and tissue collection. WH performed reverse transcription-quantitative polymerase chain reaction and gene chip array. YZ performed data analysis and drafted the manuscript. Both authors reviewed and approved the final version of the manuscript.

\section{Ethics approval and consent to participate}

The animal experiment of the present study was approved by the Ethics Committee for Use of Experimental Animals in Zhejiang Chinese Medical University (Hangzhou, China); animal permit code, SYXK (Zhejiang Province 2008-0115).

\section{Patient consent for publication}

Not applicable.

\section{Competing interests}

All authors declare that they have no competing interests. 


\section{References}

1. Aletaha D, Neogi T, Silman AJ, Funovits J, Felson DT, Bingham CO III, Birnbaum NS, Burmester GR, Bykerk VP, Cohen MD, et al: 2010 Rheumatoid arthritis classification criteria: An American college of rheumatology/European league against rheumatism collaborative initiative. Arthritis Rheum 62 : $2569-2581,2010$

2. Marrelli A, Cipriani P, Liakouli V, Carubbi F, Perricone C, Perricone R and Giacomelli R: Angiogenesis in rheumatoid arthritis: A disease specific process or a common response to chronic inflammation? Autoimmun Rev 10: 595-598, 2011

3. Kong X, Zhang Y, Liu C, Guo W, Li X, Su X, Wan H, Sun Y and Lin N: Anti-angiogenic effect of triptolide in rheumatoid arthritis by targeting angiogenic cascade. PLoS One 8: e77513, 2013.

4. Klareskog L, Catrina AI and Paget S: Rheumatoid arthritis. Lancet 373: 659-672, 2009.

5. The Chinese herbal remedy Tripterygium wilfordii Hook F in the treatment of rheumatoid arthritis. Ann Intern Med 151: I-36, 2009.

6. Goldbach-Mansky R, Wilson M, Fleischmann R, Olsen N, Silverfield J, Kempf P, Kivitz A, Sherrer Y, Pucino F, Csako G, et al: Comparison of Tripterygium wilfordii Hook F versus sulfasalazine in the treatment of rheumatoid arthritis: A randomized trial. Ann Intern Med 151: 229-240, W49-W51, 2009

7. Lu T, Zong M, Fan S, Lu Y, Yu S and Fan L: Thioredoxin 1 is associated with the proliferation and apoptosis of rheumatoid arthritis fibroblast-like synoviocytes. Clin Rheumatol 37: $117-125,2018$

8. Fan D, He X, Bian Y, Guo Q, Zheng K, Zhao Y, Lu C, Liu B, $\mathrm{Xu} \mathrm{X}$, Zhang $\mathrm{G}$ and $\mathrm{Lu} \mathrm{A}$ : Triptolide modulates TREM-1 signal pathway to inhibit the inflammatory response in rheumatoid arthritis. Int J Mol Sci 17: 498, 2016.

9. Liu C, Zhang Y, Kong X, Zhu L, Pang J, Xu Y, Chen W, Zhan H, $\mathrm{Lu} \mathrm{A}$ and Lin N: Triptolide prevents bone destruction in the collagen-induced arthritis model of rheumatoid arthritis by targeting RANKL/RANK/OPG signal pathway. Evid Based Complement Alternat Med 2013: 626038, 2013.

10. Lin N, Liu C, Xiao C, Jia H, Imada K, Wu H and Ito A: Triptolide, a diterpenoid triepoxide, suppresses inflammation and cartilage destruction in collagen-induced arthritis mice. Biochem Pharmacol 73: 136-146, 2007.

11. Chen BJ: Triptolide, a novel immunosuppressive and anti-inflammatory agent purified from a Chinese herb Tripterygium wilfordii Hook F. Leuk Lymphoma 42: 253-265, 2001.

12. Glehr M, Fritsch-Breisach M, Lohberger B, Walzer SM, Moazedi-Fuerst F, Rinner B, Gruber G, Graninger W, Leithner A and Windhager R: Influence of resveratrol on rheumatoid fibroblast-like synoviocytes analysed with gene chip transcription Phytomedicine 20: 310-318, 2013.

13. Li ZJ, Wang DQ, Hu SP, Wang XF, He Y and Zhang ZJ: A gene chip study of 'Jun Du Yan Bingzhi' on liver in a sepsis model of rat. Zhongguo Wei Zhong Bing Ji Jiu Yi Xue 21: 44-47, 2009 (In Chinese)

14. Collins JF: Gene chip analyses reveal differential genetic responses to iron deficiency in rat duodenum and jejunum. Biol Res 39: 25-37, 2006.

15. Saeki Y, Matsui T, Saisho K and Tohma S: Current treatments of rheumatoid arthritis: From the 'NinJa' registry. Expert Rev Clin Immunol 8: 455-465, 2012.

16. Wang Y, Wei D, Lai Z and Le Y: Triptolide inhibits CC chemokines expressed in rat adjuvant-induced arthritis. Int Immunopharmacol 6: 1825-1832, 2006.

17. Yifan W, Dengming W, Zheng L, Yanping L and Junkan S: Triptolide inhibits CCR5 expressed in synovial tissue of rat adjuvant-induced arthritis. Pharmacol Rep 59: 795-799, 2007.

18. Li S, Chen JW, Xie X, Tian J, Deng C, Wang J, Gan HN and Li F: Autophagy inhibitor regulates apoptosis and proliferation of synovial fibroblasts through the inhibition of PI3K/AKT pathway in collagen-induced arthritis rat model. Am J Transl Res 9: 2065-2076, 2017

19. Xiao C, Zhou J, He Y, Jia H, Zhao L, Zhao N and Lu A: Effects of triptolide from Radix Tripterygium wilfordii (Leigongteng) on cartilage cytokines and transcription factor NF-kappaB: A study on induced arthritis in rats. Chin Med 4: 13, 2009.
20. Jiao Y, Ding H, Huang S, Liu Y, Sun X, Wei W, Ma J and Zheng F: Bcl-XL and Mcl-1 upregulation by calreticulin promotes apoptosis resistance of fibroblast-like synoviocytes via activation of PI3K/Akt and STAT3 pathways in rheumatoid arthritis. Clin Exp Rheumatol 36: 841-849, 2018

21. Zhou J, Xiao C, Zhao L, Jia H, Zhao N, Lu C, Yang D, Tang JC, Chan AS and Lu AP: The effect of triptolide on CD4+ and CD8+ cells in Peyer's patch of SD rats with collagen induced arthritis. Int Immunopharmacol 6: 198-203, 2006.

22. Evans SJ, Datson NA, Kabbaj M, Thompson RC, Vreugdenhil E, De Kloet ER, Watson SJ and Akil H: Evaluation of affymetrix gene chip sensitivity in rat hippocampal tissue using SAGE analysis. Serial analysis of gene expression. Eur J Neurosci 16: 409-413, 2002

23. Wang J, Jiang Z, Ji J, Wang X, Wang T, Zhang Y, Tai T, Chen M, Sun L, Li X and Zhang L: Gene expression profiling and pathway analysis of hepatotoxicity induced by triptolide in Wistar rats. Food Chem Toxicol 58: 495-505, 2013.

24. Wang J, Wang A, Zeng $\mathrm{H}$, Liu L, Jiang W, Zhu Y and Xu Y: Effect of triptolide on T-cell receptor beta variable gene mRNA expression in rats with collagen-induced arthritis. Anat Rec (Hoboken) 295: 922-927, 2012.

25. Qu Y, Wu J, Deng JX, Zhang YP, Liang WY, Jiang ZL, Yu QH and Li J: MicroRNA-126 affects rheumatoid arthritis synovial fibroblast proliferation and apoptosis by targeting PIK3R2 and regulating PI3K-AKT signal pathway. Oncotarget 7: 74217-74226, 2016.

26. Wang M, Chen DQ, Chen L, Liu D, Zhao H, Zhang ZH, Vaziri ND, Guo Y, Zhao YY and Cao G: Novel RAS inhibitors poricoic acid ZG and poricoic acid ZH attenuate renal fibrosis via a Wnt/ $\beta$-catenin pathway and targeted phosphorylation of smad3 signaling. J Agric Food Chem 66: 1828-1842, 2018.

27. Ramirez-Bello J, Cadena-Sandoval D, Fragoso JM, Barbosa-Cobos RE, Moreno-Eutímio MA, Saavedra-Salinas MÁ, Valencia-Pacheco G,López-Villanueva RF and Jiménez-Morales S: The VEGFA-1154G/A polymorphism is associated with reduced risk of rheumatoid arthritis but not with systemic lupus erythematosus in Mexican women. J Gene Med 20: e3024, 2018.

28. Murayama MA, Kakuta S, Maruhashi T, Shimizu K, Seno A, Kubo S, Sato N, Saijo S, Hattori M and Iwakura Y: CTRP3 plays an important role in the development of collagen-induced arthritis in mice. Biochem Biophys Res Commun 443: 42-48, 2014.

29. Salliot $C$ and van der Heijde D: Long-term safety of methotrexate monotherapy in patients with rheumatoid arthritis: A systematic literature research. Ann Rheum Dis 68: 1100-1104, 2009.

30. Malemud CJ: Negative regulators of JAK/STAT signaling in rheumatoid arthritis and osteoarthritis. Int J Mol Sci 18: 484, 2017.

31. Wang J, Li Y, Yang Y, Du J, Zhao M, Lin F, Zhang S and Wang B: Systems pharmacology dissection of multiscale mechanisms of action for herbal medicines in treating rheumatoid arthritis. Mol Pharm 14: 3201-3217, 2017.

32. Tu S, Hu Y, Zeng K, Zhang M, Lai X and Weichen Z: Effects of triptolide on the expression and activity of NF-kappaB in synovium of collagen-induced arthritis rats. J Huazhong Univ Sci Technolog Med Sci 25: 543-545, 2005.

33. Matta R, Wang X, Ge H, Ray W, Nelin LD and Liu Y: Triptolide induces anti-inflammatory cellular responses. Am J Transl Res 1: 267-282, 2009.

34. Yang M, Huang J, Pan HZ and Jin J: Triptolide overcomes dexamethasone resistance and enhanced PS-341-induced apoptosis via PI3k/Akt/NF-kappaB pathways in human multiple myeloma cells. Int J Mol Med 22: 489-496, 2008.

35. Liu YY, Jiao ZY, Li W and Tian Q: PI3K/AKT signaling pathway activation in a rat model of migraine. Mol Med Rep 16: 4849-4854, 2017

36. Clarimundo VS, Farinon M, Pedó RT, Teixeira VON, Nör C, Gulko PS, Xavier RM and de Oliveira PG: Gastrin-releasing peptide and its receptor increase arthritis fibroblast-like synoviocytes invasiveness through activating the PI3K/AKT pathway. Peptides 95: 57-61, 2017.

This work is licensed under a Creative Commons Attribution-NonCommercial-NoDerivatives 4.0 International (CC BY-NC-ND 4.0) License. 